\title{
Erratum to: Exposure and Risk Assessment of Insecticide Methomyl for Applicator during Treatment on Apple Orchard
}

Eunhye Kim · Joon-Kwan Moon · Hoon Choi - Su-Myoung Hong •

Dong-Hyuk Lee $\cdot$ Hyomin Lee $\cdot$ Jeong-Han Kim

Published online: 29 February 2012

(B) Springer-Verlag 2013

Erratum to: J Korean Soc Appl Biol Chem DOI 10.1007/s13765-012-0016-1

Page 99 Third paragraph, right column. The last sentence of the paragraph "If MOS is $<1$, the working condition is considered to be low risk" is incorrect.

The sentence should read follows:

"If MOS is $<1$, the working condition is considered to be unsafe." 